University of Nebraska - Lincoln

DigitalCommons@University of Nebraska - Lincoln

\title{
8-15-1989
}

\section{THE CHROMOSPHERE OF BETA CASSIOPEIAE}

\author{
Terry J. Teays \\ University of Nebraska-Lincoln, tteays1@jhu.edu \\ Edward G. Schmidt \\ University of Nebraska-Lincoln, eschmidt1@unl.edu \\ Massimo Fracassini \\ Dipartimento di Fisica, Universita degli Studi di Milano \\ Laura E. Pasinetti Fracassini \\ Dipartimento di Fisica, Universita degli Studi di Milano
}

Follow this and additional works at: https://digitalcommons.unl.edu/physicsschmidt

Part of the Physics Commons

Teays, Terry J.; Schmidt, Edward G.; Fracassini, Massimo; and Pasinetti Fracassini, Laura E., "THE CHROMOSPHERE OF BETA CASSIOPEIAE" (1989). Edward Schmidt Publications. 23.

https://digitalcommons.unl.edu/physicsschmidt/23

This Article is brought to you for free and open access by the Research Papers in Physics and Astronomy at DigitalCommons@University of Nebraska - Lincoln. It has been accepted for inclusion in Edward Schmidt Publications by an authorized administrator of DigitalCommons@University of Nebraska - Lincoln. 
ThE ASTROPHYSICAL JoURNAL, 343:916-919, 1989 August 15

(1) 1989. The American Astronomical Society. All rights reserved. Printed in U.S.A.

\title{
THE CHROMOSPHERE OF BETA CASSIOPEIAE
}

\author{
TERRY J. TEAYS ${ }^{1,2}$ \\ Behlen Observatory, Department of Physics and Astronomy, University of Nebraska-Lincoln, \\ and Astronomy Programs Computer Sciences Corporation \\ EDWARD G. SCHMIDT \\ Behlen Observatory, Department of Physics and Astronomy, University of Nebraska-Lincoln \\ AND \\ Massimo Fracassini and Laura E. PasinetTi Fracassini \\ Dipartimento di Fisica, Universitá degli Studi di Milano \\ Received 1988 October 3; accepted 1989 January 31
}

\begin{abstract}
We have carried out high-resolution, long-wavelength IUE observations and ground-based photometry of the $\delta$ Scuti star $\beta$ Cas. The ground-based observations were used together with previous results of Antonello et al. to ensure that the $I U E$ observations were correctly phased relative to the photometric variation. Fluxes for the emission core of the Mg II $k \lambda 2796$ line were obtained from the ultraviolet spectra over several cycles in 1986 and 1987. We found that there is an increase in the emission during part of the cycle but it occurs near minimum light in contrast to another $\delta$ Scuti star, $\rho$ Pup, and the classical Cepheids where it occurs near maximum light. The mean level of chromospheric activity is comparable to other early $F$ dwarfs.

Subject headings: stars: chromospheres - stars: Delta Scuti — stars: individual ( $\beta$ Cas) ultraviolet: spectra
\end{abstract}

\section{INTRODUCTION}

Fracassini and Pasinetti (1982) surveyed $\delta$ Scuti variables for chromospheric emission in the $\mathrm{Mg}$ II $h$ and $k$ lines. Even though they observed each star at only one phase of its variation, emission was clearly present in three of the stars and was possibly present in three others. This result supported the earlier studies of Le Contel et al. (1970) and Dravins, Lind, and Sarg (1977) in which weak chromospheric emission was found in the cores of the Ca II $K$ line for the $\delta$ Scuti stars $\gamma$ Boo and $\rho$ Pup.

The discovery of chromospheric activity in the lowest luminosity Cepheid strip stars suggests a parallel with the activity which has been observed in the classical Cepheids (Kraft 1957; Schmidt and Parsons 1984). The activity in those stars was found to be dependent on the phase of the pulsation, and Schmidt and Parsons suggested that the pulsation has a significant influence on the chromosphere. In addition, the $\mathbf{M g}$ II emission lines for the classical Cepheids indicated geometrically deep chromospheric regions and large-velocity gradients.

Subsequently, Fracassini et al. (1983) studied a series of spectra covering one and a half cycles of $\rho$ Pup, one of the stars from the survey with obvious emission. As expected, the strength of the emission varied through the cycle with a peak near maximum light (see their Fig. $3 b$ ). For an interval around minimum light the emission is relatively constant but is always present.

Garbuzov and Andrievskii (1986) discussed the excitation of chromospheric emission in $\delta$ Scuti stars and concluded that heating by a pulsation-generated shock could explain the observed behavior.

\footnotetext{
${ }^{1}$ Guest Observer with the International Ultraviolet Explorer satellite.

${ }^{2}$ Staff member of the International Ultraviolet Explorer Observatory, at the Laboratory for Astronomy and Solar Physics, N 4SA Goddard Space Flight Center.
}

In the present paper we discuss ultraviolet spectra for the star $\beta$ Cas. This star was discovered to be a $\delta$ Scuti star by Millis (1966). It has a period of a tenth of a day and a spectral type of F2 III-IV. Although it is somewhat hotter than $\rho$ Pup (F6 IIp), which reduces the contrast between the photosphere and the chromospheric emission, Kondo et al. (1972), Ayres, Marstad, and Linsky (1981) and Fracassini and Pasinetti (1982) reported $\mathrm{Mg}$ II emission. It is therefore a good candidate for more detailed study.

Beta Cas is listed as a spectroscopic binary in the Bright Star Catalog and elsewhere based on a study by Mellor (1917). However, more recently Abt (1965) and Yang, Walker, and Fahlman (1982) concluded that there is no evidence to confirm that result. We will therefore consider $\beta$ Cas to be a single star.

\section{THE OBSERVATIONS}

\section{a) IUE Observations}

We obtained two series of long-wavelength, high-resolution IUE spectra of $\beta$ Cas. The first spanned an interval of $5.5 \mathrm{hr}$ (2.2 cycles) on 1986 September $2 / 3$ (Julian Date $2,446,676-7$ ), while a second series on 1987 November 11 (Julian Date $2,447,111)$ was exposed over nearly three cycles. A single spectrum was also obtained on 1987 August 19 (Julian Date $2,447,027)$. The spectra are listed in Table 1 with the Heliocentric Julian Date, the phase in the pulsation cycle, and fluxes discussed below. In calculating the phases we used a period of 0.10101 days (Antonello, Pastori, and Poretti 1986). For the data from 1986 the epoch of maximum light was taken to be H.J.D. $=2,446,704.557$ (E. Antonello, private communication) while for the data of 1987 we used the epoch H.J.D. = $2,447,119.117$ which is discussed below. Although the exposure times, 1-2 minutes, are short compared to the period, the gaps between exposures imposed by the readout time of the camera amount to about a quarter of a cycle. This restricted the sampling during any one cycle but having covered more than two 
TABLE 1

EXPOSURE LOG

\begin{tabular}{|c|c|c|c|}
\hline $\begin{array}{l}I U E \\
\text { Image No. } \\
\text { LWP }\end{array}$ & $\begin{array}{c}\text { HJD -- } \\
2,440,000\end{array}$ & $\begin{array}{l}\text { Phase } \\
\text { in Cycle }\end{array}$ & $\begin{array}{c}\mathrm{Mg} \mathrm{II} k \\
\text { Flux } \\
10^{-11} \mathrm{ergs} \mathrm{cm}^{-2} \mathrm{~s}^{-1}\end{array}$ \\
\hline $8996 \ldots$ & 6676.8213 & 0.42 & 2.3 \\
\hline $8997 \ldots$ & 6676.8481 & 0.68 & 0.4 : \\
\hline $8998 \ldots$ & 6676.8719 & 0.92 & 0.8 : \\
\hline $8999 \ldots$ & 6676.8962 & 0.16 & 0 \\
\hline $9000 \ldots$ & 6676.9259 & 0.45 & 2.4 \\
\hline $9001 \ldots$ & 6676.9657 & 0.85 & 1.7: \\
\hline $9002 \ldots$ & 6676.9901 & 0.09 & 0.4 : \\
\hline $9003 \ldots \ldots \ldots \ldots \ldots$ & 6677.0167 & 0.35 & 0.3 \\
\hline $9004 \ldots \ldots \ldots \ldots \ldots$ & 6677.0442 & 0.62 & 0.6 \\
\hline $11431 \ldots$ & 7027.1161 & 0.19 & 0.2 \\
\hline $12067 \ldots$ & 7111.6478 & 0.06 & 0.1 : \\
\hline $12068 \ldots$ & 7111.6826 & 0.40 & 0 \\
\hline $12069 .$. & 7111.7077 & 0.65 & 0 \\
\hline $12070 .$. & 7111.7303 & 0.87 & 0.2 \\
\hline $12071 \ldots$ & 7111.7554 & 0.12 & 0.2 \\
\hline $12072 \ldots$ & 7111.7797 & 0.36 & 0 \\
\hline $12073 \ldots$ & 7111.8054 & 0.62 & 0 \\
\hline 12074 .. & 7111.8284 & 0.84 & 0.1 \\
\hline $12075 \ldots$ & 7111.8514 & 0.07 & 0.1 \\
\hline $12076 \ldots$ & 7111.8742 & 0.30 & 0 \\
\hline $12077 \ldots \ldots$ & 7111.8971 & 0.52 & 1.4 \\
\hline $12078 \ldots \ldots \ldots \ldots \ldots$ & 7111.9205 & 0.76 & 0.2 \\
\hline $12079 \ldots$ & 7111.9475 & 0.02 & 0.1 \\
\hline
\end{tabular}

cycles in each of the two series of observations we obtained good phase sampling.

The spectra were used to study the $\mathrm{Mg}$ II $h$ and $k$ emission cores. Obtaining the strength of emission features requires some type of fitting of the underlying photospheric absorption line. Some investigators have chosen to include the entire intensity between the edges of the emission while others have fitted a profile to the absorption line. We have chosen another method which is suited to this star but not necessarily of general utility. Since $\beta$ Cas exhibits a small amplitude, we expect that the underlying photospheric profile should exhibit little change. Furthermore, our initial examination of the spectra showed that the emission is weak at best and mostly absent. Thus we have formed a mean profile from all 23 of the spectra and used that as a base line from which any emission was measured. Examples of a profile for which we find no emission and the profile with the largest emission are plotted in Figure 1 along with the mean profile. It can be seen that the emission is certainly significant in the latter while the former scatters about the mean profile.

An examination of Figure 1 shows that in LWP 9000, the spectrum with the strongest emission, the emission was about $30 \%$ above the mean profile. This emission together with that from the other three spectra with emission fluxes exceeding $10^{-11} \mathrm{ergs} \mathrm{cm}^{-2} \mathrm{~s}^{-1}$ would have raised the mean profile by only $4 \%$ when averaged with the 19 other spectra. Since this is less than the noise in the spectra we did not form a new profile with those spectra omitted. Doing so would produce differences in the numbers in Table 1 which are less than the random errors.

Table 1 lists the fluxes of the $\mathrm{Mg} k$ line for all of the spectra. The $\mathrm{Mg} h$ line is weaker in emission and the results less certain so we have not tabulated those results. In Figure 2 we have plotted the $\mathrm{Mg}$ II $k$ line fluxes against the phase. Little emission is present except during an interval around phase 0.45 .

\section{b) Ground-Based Photometry}

Because of the short period of this star, 4300 cycles elapsed between the two series of $I U E$ observations. Since the period is not accurate enough to reliably extrapolate the time of maximum light over such a long interval, it is desirable to determine an epoch of maximum light for each year. For the 1986 observations we used the epoch and period derived by Antonello et al. and E. Antonello (private communication) from photometry which was contemporaneous with our IUE observations. One of us (E. G. S.) undertook ground-based observations during 1987 November to obtain an epoch of maximum light for that year.

$\mathrm{V}$ band observations were made for $4 \frac{3}{4} \mathrm{hr}$ on 1987 November 20 and $3 \mathrm{hr}$ on 1987 November 21 with the CCD photometer on the Behlen Observatory $76 \mathrm{~cm}$ telescope. The instrument and reduction procedures are described by Schmidt (1988). A mask was placed over the telescope to restrict the aperture due to the brightness of the star. Since there was no suitable comparison star in the field of the CCD, the telescope was moved back and forth between $\beta$ Cas and the comparison star, HR 60 .

An examination of the data indicated that the conditions were photometric over all except about 40 minutes of the interval (which was omitted) and a total of 96 individual differential magnitudes were available. A sine curve of full amplitude 0.027 magnitudes with maximum light at H.J.D. $=2,447,119.117$ was found to fit the data. The scatter about the fitted sine wave, $\pm 0.017 \mathrm{mag}$, is typical of individual differential magnitudes obtained at Behlen Observatory on photometric nights. The amplitude agrees well with the value of $0.03 \mathrm{mag}$ found a year earlier by Antonello et al.

\section{DISCUSSION}

Referring to Figure 2, we see a phase-dependent variation of the flux. A spike of emission is evident between phases 0.4 and 0.6 and the fluxes are otherwise small. The data from 1985 and 1986 are consistent with the possible exception of the high point at phase 0.84 and the slightly high point at phase 0.92 . Since these points were derived from exceptionally noisy spectra and are thus marked as uncertain in Table 1 and since other data near those phases lack significant emission, they are unlikely to indicate the presence of an additional episode of emission.

The emission fluxes we have detected are small and their reality might be questioned. However, an examination of Figure 1 shows that at its strongest, the emission in both $\mathrm{Mg}$ II $h$ and $\mathrm{Mg}$ II $k$ is significantly above the mean profile. In addition the consistency of the behavior of the fluxes when plotted against phase argues that the increased flux in the middle of the cycle is a real effect.

In $\rho$ Pup Fracassini et al. (1983) also observed variations in the emission strength. However, they found that the maximum of the emission occurred at maximum light, a behavior similar to that seen in classical Cepheids (Schmidt and Parsons 1984) where the peak of the Mg II emission was observed during rising light. Thus, the phasing of the emission in $\beta$ Cas is a surprising result which must be explained in terms of some difference in the dynamics of the pulsation of this star compared with $\rho$ Pup.

The mean emission level over the cycle is $0.5 \times 10^{-11}$ ergs $\mathrm{cm}^{-2} \mathrm{~s}^{-1}$. There is little evidence of differences between the observations of the two seasons which is consistent with the 


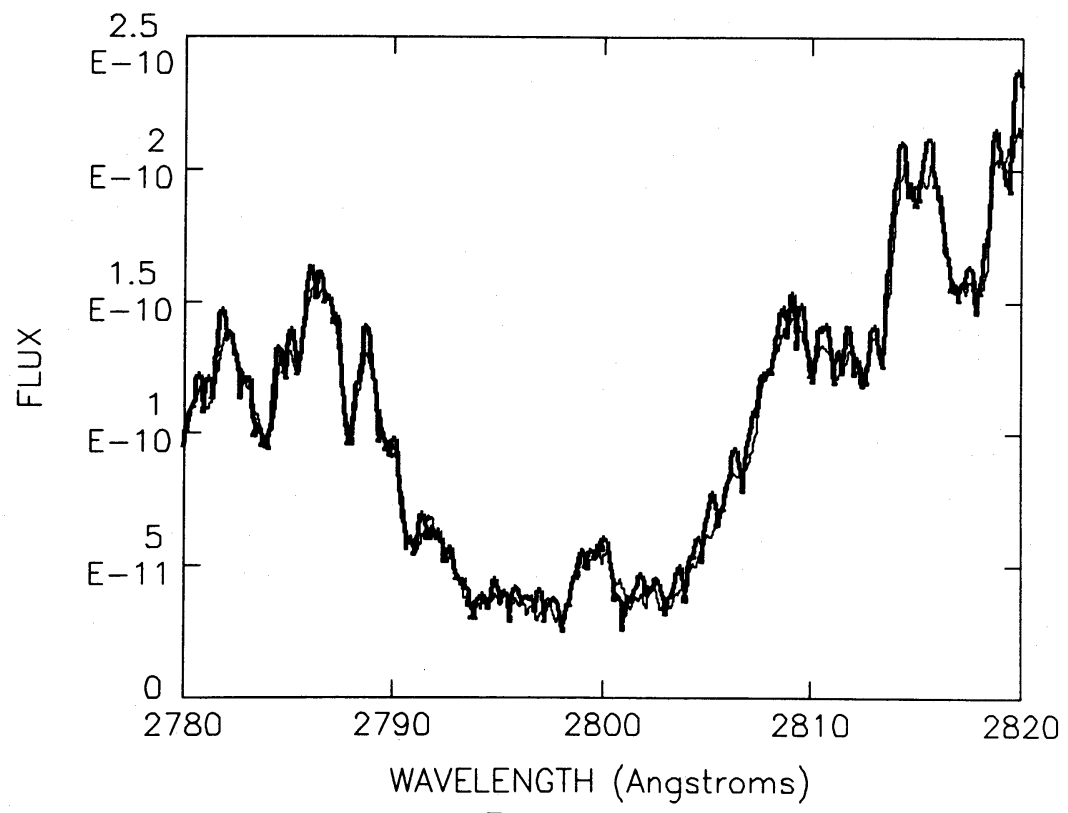

FIG. $1 a$

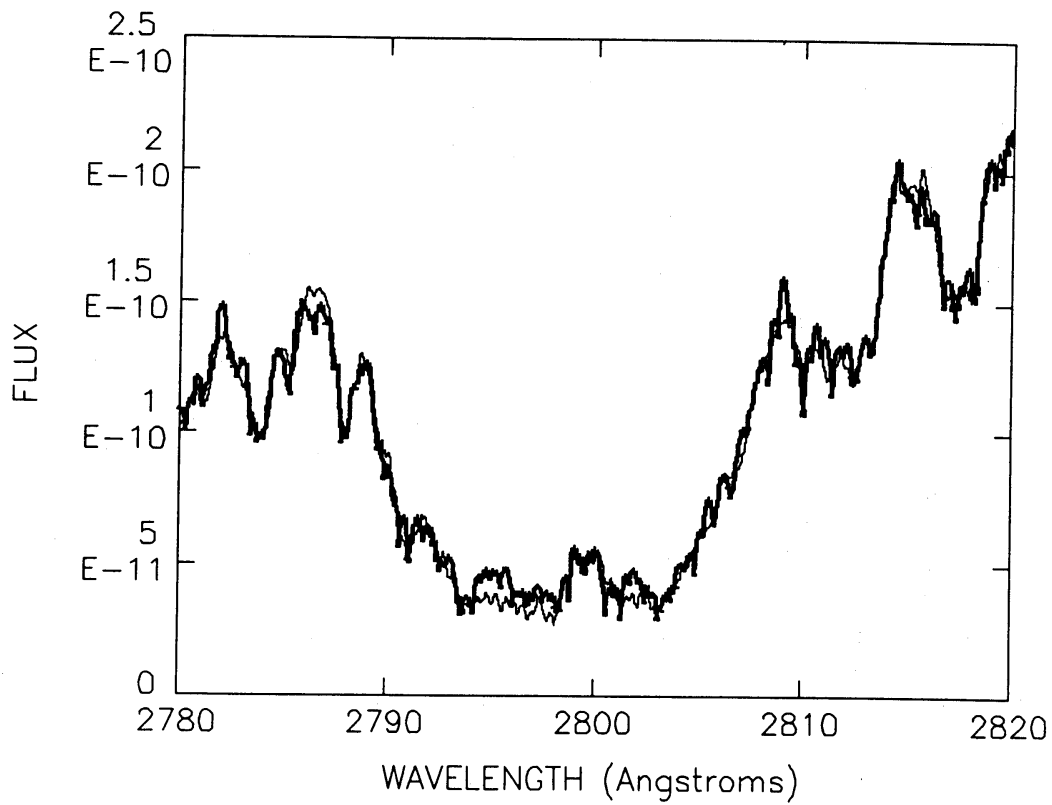

FIG. $1 b$

Fig. 1.- Spectra in the region of the $\mathrm{Mg}$ II lines at 22900 . The mean profile from all of the spectra is shown as the light line in each case and the dark lines depict the individual spectra from frames (a) LWP 8999 and $(b)$ LWP 9000 . The former exhibits no significant emission while the latter has the strongest emission of any of our spectra.

fact that the amplitude in visible light of $\beta$ Cas was the same in 1986 and 1987.

To compare $\beta$ Cas with other early $\mathrm{F}$ dwarfs we have calculated the flux in terms of the stellar luminosity. For this we used an apparent bolometric flux of $3.0 \times 10^{-6} \mathrm{ergs} \mathrm{cm}^{-2} \mathrm{~s}^{-1}$ (Ayres et al. 1981). The mean Mg II $k$ flux of $\beta$ Cas is then $17 \times 10^{-7}$ of the luminosity while the peak emission is $80 \times 10^{-7}$ of the luminosity. As discussed above, we have taken our fluxes above the mean absorption profile. A $2 \AA$ band (the approximate width of the emission feature) at the minimum of our mean profile contains a flux of $7.2 \times 10^{-11}$ ergs $\mathrm{cm}^{-2} \mathrm{~s}^{-1}$. To make our fluxes compatible with published values in which the entire intensity at the line core was used, this should be added. We then obtain a mean $\mathrm{Mg}$ II $k$ flux of $260 \times 10^{-7}$ and a peak value of $320 \times 10^{-7}$.

Ayres et al. list $\mathrm{Mg}$ II emission fluxes for five stars, including $\beta$ Cas, with spectral types of F2, F3, or F5 and luminosity classes of V or IV. Their fluxes include both the $h$ and the $k$ line so we should approximately double our values to compare with theirs. The value they give for $\beta$ Cas, for which we do not know the phase, is $230 \times 10^{-7}$ while the other $\mathrm{F}$ dwarfs have emission fluxes ranging from $100 \times 10^{-7}$ to $290 \times 10^{-7}$ of the bolometric flux. Kondo et al. (1976) give a similar value, $300 \times 10^{-7}$, for $\beta$ Cas. Thus our emission-line fluxes are about twice as large as the previous values for similar stars. However, the correction for the different handling of the residual inten- 


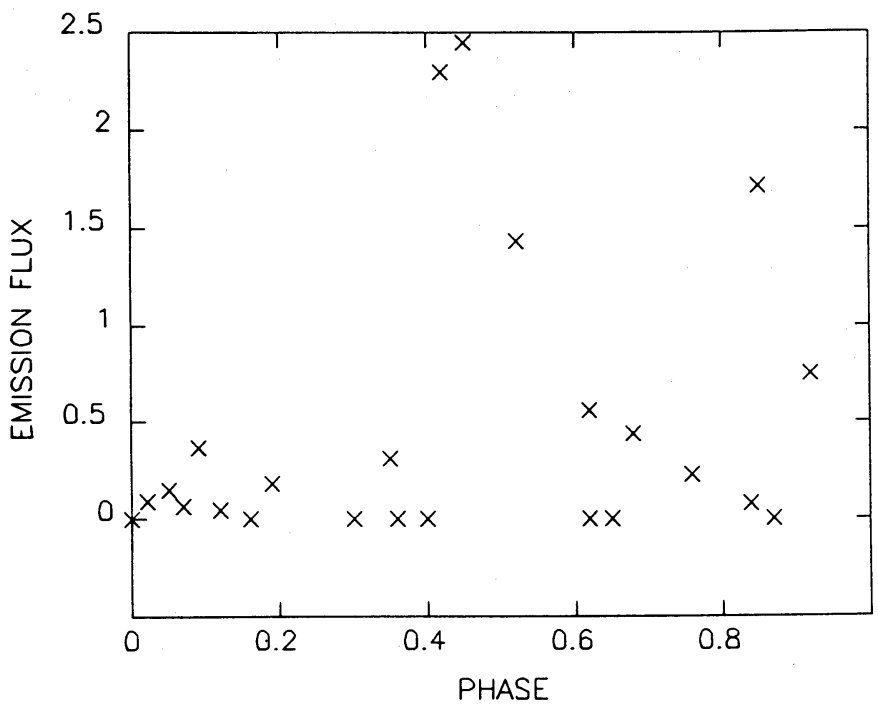
phase

FIG. 2.-The strength of the $\mathrm{Mg}$ II $k \lambda 2796$ emission core plotted against the

sity in the center of the absorption line increased our mean flux by a factor of 15 and the peak flux by a factor of 4 . Clearly, a reanalysis of the data for $\beta$ Cas or for the other stars is needed to make the handling of this problem consistent before we can say that the fluxes in $\beta$ Cas are significantly higher than for other stars. We conclude that there is no reason to believe that the chromosphere of $\beta$ Cas is greatly different than that of other stars of similar temperature and gravity.

\section{CONCLUSIONS}

1. Chromospheric activity in $\beta$ Cas is modulated by the pulsation.

2. In $\beta$ Cas the chromospheric activity is observed to occur near minimum light. This contrasts with the behavior observed in another $\delta$ Scuti star, $\rho$ Pup, and in the classical Cepheids where the emission is observed near maximum light.

3. The mean levels of activity are comparable to other early F dwarfs.

We are grateful to the staffs of the International Ultraviolet Explorer Observatory and the University of Colorado Regional Data Analysis Facility for their assistance in obtaining and analyzing the data used in this paper. We also thank Catherine Imhoff for very useful suggestions regarding the extraction of emission fluxes from the spectra. This work was supported by the National Aeronautics and Space Administration through the use of the International Ultraviolet Explorer Observatory and grant number NAG 5-839.
Abt, H. A. 1965, Ap. J. Suppl., 11, 429.

Ayres, T. R., Marstad, N. C., and Linsky, J. L. 1981, Ap. J., 247, 545.

Antonello, E., Pastori, L., and Poretti, E. 1986, Inf. Bull. Var. Stars, No. 2958.

Dravins, D., Lind, J., and Sarg, K. 1977, Astr. Ap., 54, 381.

Fracassini, M., and Pasinetti, L. E. 1982, Astr. Ap., 107, 326.

Fracassini, M., Pasinetti, L. E., Castelli, F., Antonello, E., and Pastori, L. 1983,

Ap. Space Sci., $97,323$.

Garbuzov, G. A., and Andrievskii, S. M. 1986, Astrofizika, 25, 251.

Kondo, Y., Duval, J. E., Modisette, J. L., and Morgan, T. H. 1976, Ap. J., 210, 713.

Kondo, Y., Giuli, R. T., Modisette, J. L., and Rydgren, A. E. 1972, Ap. J., 176, 153.

\section{FRACASSINI: deceased}

L. E. Pasinetri FraCassini: Universitá degli Studi di Milano, Dipartimento di Fisica, Via Celoria 16, 20133 Milano, Italy

E. G. SCHMIDT: Department of Physics and Astronomy, University of Nebraska, Lincoln, NE 68588-0111

T. J. TEAys: Code 684.9, IUE Observatory, Goddard Space Flight Center, Greenbelt, MD 20771
Kraft, R. P. 1957, Ap. J., 125, 336.

Le Contel, J. M., Praderie, F., Bijaoui, A., Dantel, M., Sareyan, J. P. 1970, Astr. Ap., 8, 159 .

Mellor, L L 1917, Pub. Obs. Univ. Mich, 3,61.

Millis, R. L. 1966, Pub. A.S.P., 78, 340.

Schmidt, E. G. 1988, Proc. 9th Annual Fairborn-Smithsonian-IAPPP Symp., ed. D. Hayes (Fairborn Observatory Press), in press.

Schmidt, E. G., and Parsons, S. B. 1984, Ap. J $279,202$.

Yang, S., Walker, G. A. H., and Fahlman, G. G. 1982, Pub. A.S.P., 94, 317. 